\title{
Size Effects: The Relation to the Percentage of Atoms That Participate in the Deformation of $\mathrm{ZrCu}$ Metallic Glass
}

\author{
L. K. Gao, F. L. Zhao, N. Xu, L. Qi, and R. P. Liu \\ State Key Laboratory of Metastable Materials Science \& Technology, Yanshan University, \\ Hebei Street 438, Qinhuangdao 066004, China \\ Correspondence should be addressed to L. Qi; qili@ysu.edu.cn and R. P. Liu; riping@ysu.edu.cn
}

Received 29 April 2014; Accepted 28 May 2014; Published 15 June 2014

Academic Editor: Xinqing Chen

Copyright (C) 2014 L. K. Gao et al. This is an open access article distributed under the Creative Commons Attribution License, which permits unrestricted use, distribution, and reproduction in any medium, provided the original work is properly cited.

Molecular dynamics simulations indicate that with the model diameter gradually decreasing the deformation mode of $\mathrm{ZrCu}$ metallic glass evolves from highly localized shear band formation to homogeneous deformation with obvious transition in $D=7-9 \mathrm{~nm}$. Through the statistic of atoms that sustain shear strain larger than $8 \%$ in the models with $8 \%$ strain along $z$-direction, we found that the main reason for the uniform deformation that occurs in the smallest size model is that there are $61 \%$ atoms involved in the deformation, which significantly decrease the strain assigned to individual atoms, avoiding large atomic rearrangement and making those atoms evenly distributed in the model matrix.

\section{Introduction}

Metallic glasses (MGs) are a relatively new materials that have many excellent properties, such as high strength, high elastic limit, and high corrosion resistance [1]. But they almost have no plasticity under low temperature loads; this is mainly due to the highly spontaneous localization within a narrow shear band during the plastic deformation process, which rapidly expands along the shear plane and disables the material instantaneously [2]; this defect seriously affects the MGs as the application of engineering material. So how to improve the plasticity of MGs under low temperature loads became the focus of study. Similar to the crystalline alloy, reducing the size of the amorphous alloy is a kind of possible way to improve the plasticity $[3,4]$, but there are many controversies about size effects on amorphous alloy. (i) The preparation of micron or nanometer MGs usually uses focused ion beam (FIB) in experiments, the highenergy ion beam would damage the surface [5-7] and the fabricated sample generally exists $1^{\circ}-2^{\circ}$ deviation and forms a cone, and the cone sample inevitably induces complex stress states and deformation localization on the end of the sample [8]. (ii) With the decrease of the sample size, the change of deformation behavior is gradual or abrupt due to the difficulty to preparation of nanoscale sample, making the problem hard to get a system discussion and proof in experiment. So the size effects on the deformation behavior and deformation mechanism of MGs has always been a continuous explore problem.

In this paper, we systematically study the size effects on the deformation behavior of the nanoscale MGs using molecular dynamics simulation. Molecular dynamics simulation can prepare perfect nanoscale cylindrical model, and the preparation process would not damage the surface state, so we can cleverly avoid some experiment shortages and play a supplement and guiding role in experimental study.

\section{Model and Method}

In our MD simulation, we studied the deformation behavior and the deformation mechanism of $\mathrm{ZrCu}$ metallic glass (MG) with different size models. $\mathrm{ZrCu}$ amorphous alloy is a binary alloy [9], since the binary alloys easier to model and analyse than the multielement alloys, it makes $\mathrm{ZrCu}$ an attractive MG to study theoretically. The simulations are carried out using the embedded atom method (EAM) potential, supplied in LAMMPS [10]. A small box of 10000 random configuration atoms with $3 \mathrm{D}$ periodic boundary conditions (PBCs) is first equilibrated at $2600 \mathrm{~K}$ for $50 \mathrm{ps}$, and then we cool the system from $2600 \mathrm{~K}$ down to $50 \mathrm{~K}$ in 


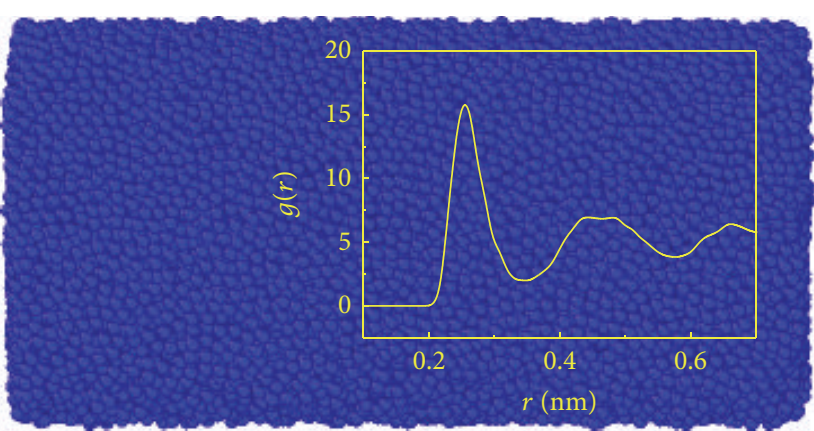

(a)

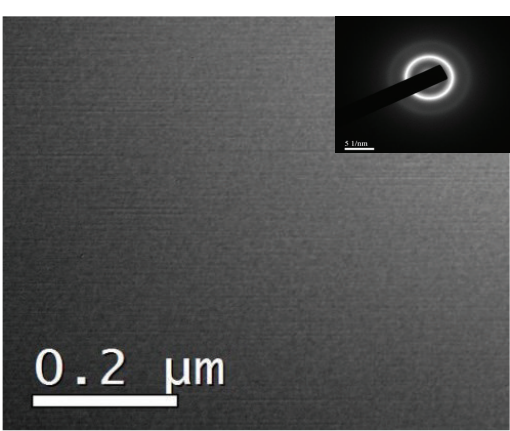

(b)

FIgUre 1: (a) The amorphous alloy model and (b) TEM photo of the $\mathrm{ZrCu}$ alloy.

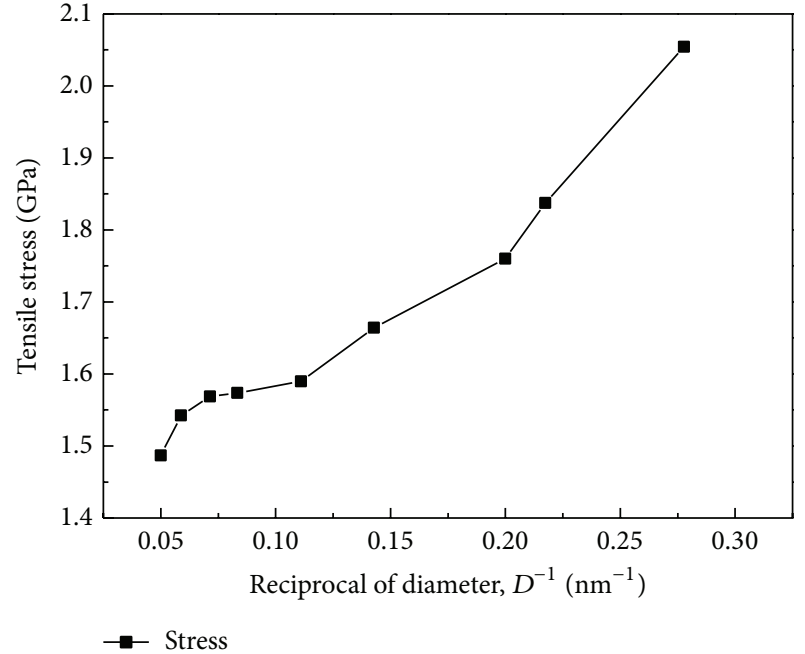

FIgURE 2: Tensile strength change curve with the decrease of the model size.

$50 \mathrm{~K}$ decrements at a constant quenching rate of $2 \mathrm{~K} / \mathrm{ps}$ to the glass state $(50 \mathrm{~K})$, at zero external pressure as shown in Figure 1. Figure 1(a) is the RDF of the simulation model which indicates the amorphous structure. Figure 1(b) shows a typical bright-field TEM image of $\mathrm{ZrCu}$ alloy and its corresponding selected-area electron diffraction (SAED) pattern. It can be seen that a uniform image without any contrast and sharp electron diffraction rings were observed, indicating a typical amorphous structure. To get large samples, we replicate the 10000 atom configuration, which then annealed for 500 ps at $800 \mathrm{~K}$ to remove the increased surface energy and then relaxation under $50 \mathrm{~K}$ for $500 \mathrm{ps}$. By deleting the redundant atoms from cube configuration we get a series of diameters (D) from $3.6 \mathrm{~nm}$ to $20 \mathrm{~nm}$ amorphous cylindrical model with aspect ratio of $2: 1$. A constant strain rate of $1 \times 10^{8} \mathrm{~s}^{-1}$ along $z$-direction is used at low temperature of $50 \mathrm{~K}$ by moving the rigid atoms at one end along the $z$ axis, while keeping the rigid atoms at the other end unchanged.

\section{Results and Discussion}

Figure 2 shows the relationship of the tensile strength and the reciprocal of diameter for different size models. We find that

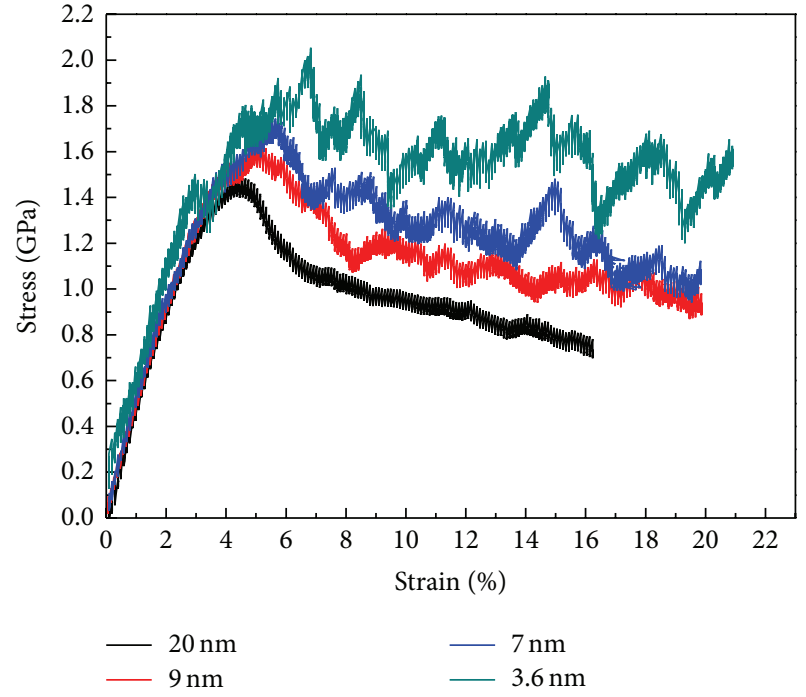

FIGURE 3: The typical stress-strain curves for different size models during the tensile process.

with the decrease of the model size the corresponding tensile strength increased significantly, and the smaller the model size the more significant increase of the tensile strength, suggesting the stronger ability to resist deformation of the model. It is worth noting that along the decreasing of model size the tensile strength is increased gradually.

Figure 3 shows the typical stress-strain curves, from which we observe that in the smallest size model the stressstrain curve shows an obvious "rise and fall" phenomenon due to the stress is sensitive to the formation and propagation of individual plastic events in the small size model. In the $D=7-9 \mathrm{~nm}$ model the wave amplitude and the number of fluctuation decreased greatly; this is mainly because with the increase of the model size, more local strain starts at the same time during the deformation process which weakens the mutation of stress and the wave amplitude of fluctuation. In the $20 \mathrm{~nm}$ cylindrical model the fluctuation phenomenon disappears completely and presents a smooth stress strain curve; however when the stress reaches its maximum then accompanied by shear softening occurs a sharply decline, 


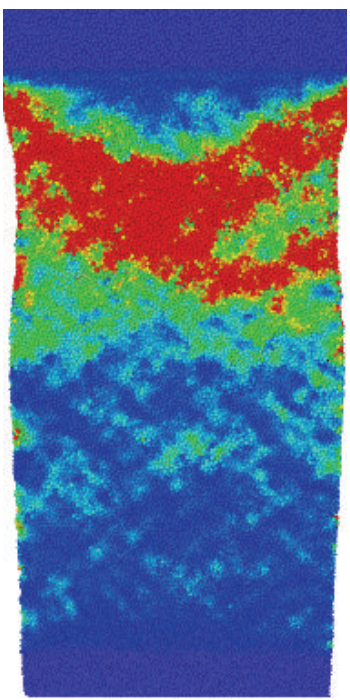

(a)

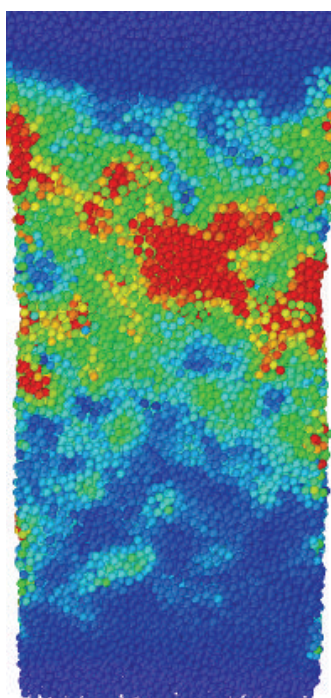

(b)

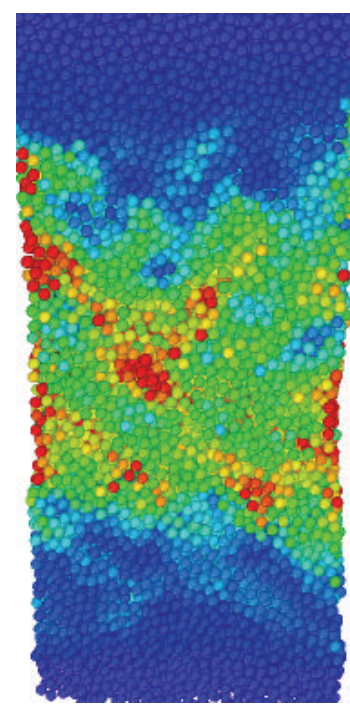

(c)

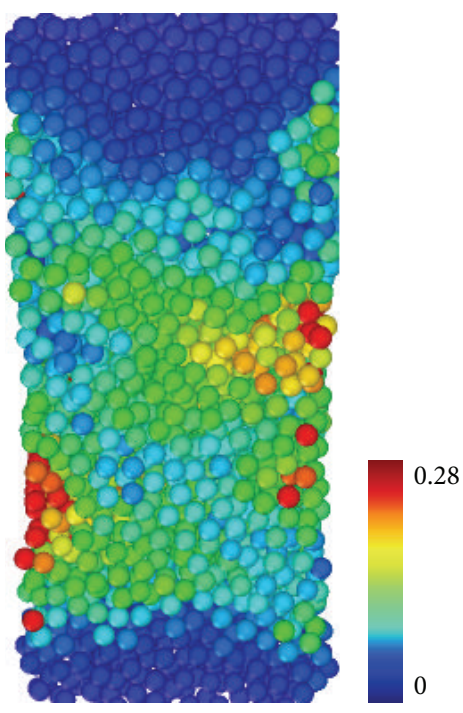

(d)

Figure 4: The distribution of local strain along the diameter: (a) $D=20 \mathrm{~nm}$, (b) $D=9 \mathrm{~nm}$, (c) $D=7 \mathrm{~nm}$, and (d) $D=3.6 \mathrm{~nm}$.

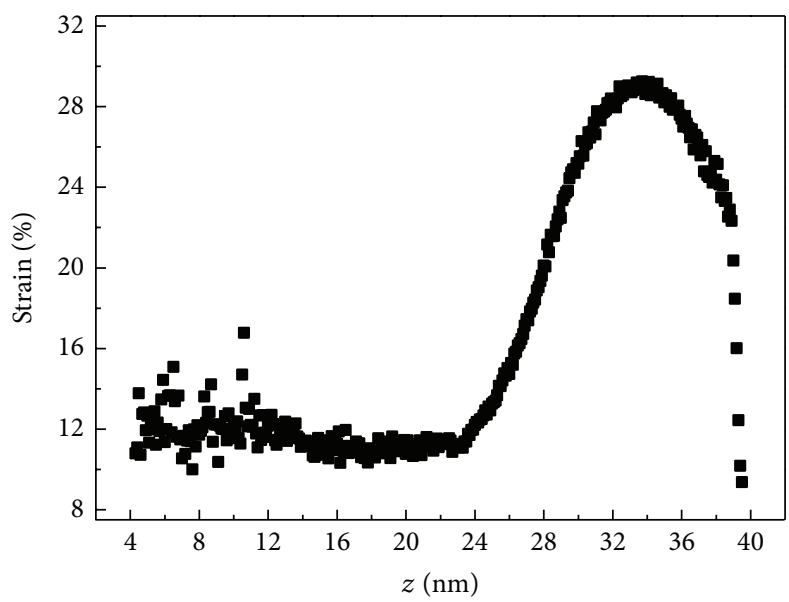

- $D=20 \mathrm{~nm}$

(a)

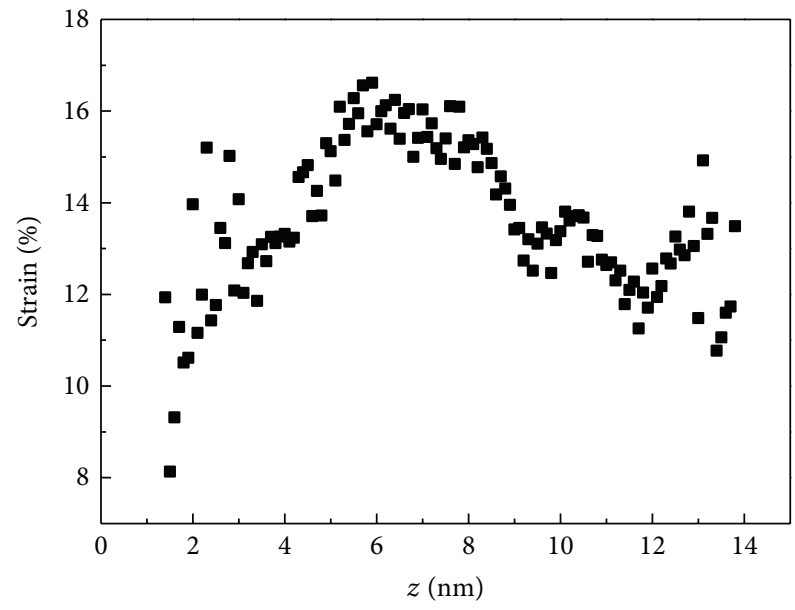

- $D=7 \mathrm{~nm}$

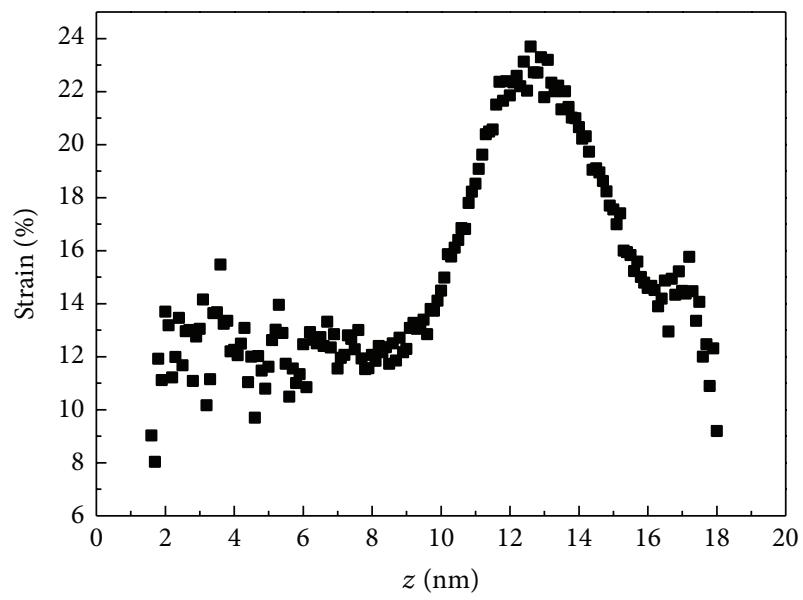

- $D=9 \mathrm{~nm}$

(b)

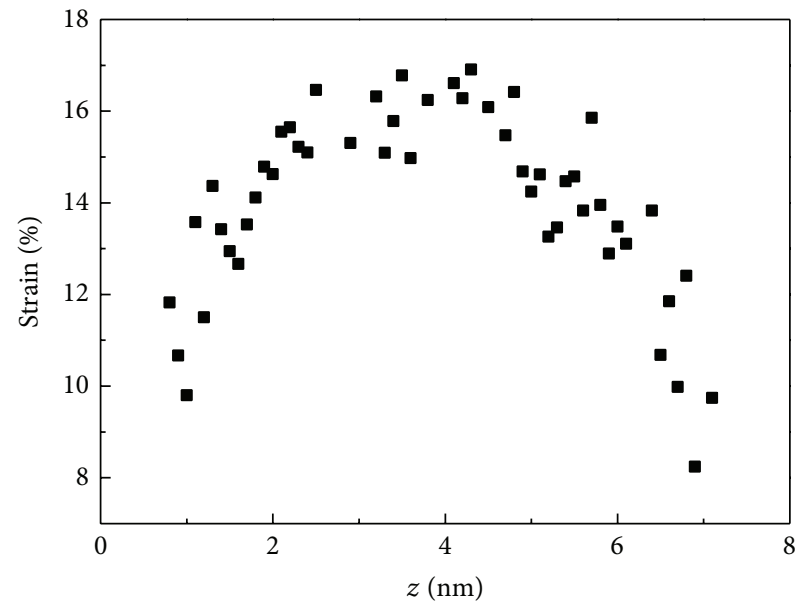

- $D=3.6 \mathrm{~nm}$

(c)

(d)

FIGURE 5: Statistical distribution of individual atom with shear strain $>8 \%$ ((a), (b), (c), and (d) are $D=20 \mathrm{~nm}, 9 \mathrm{~nm}, 7 \mathrm{~nm}$, and $3.6 \mathrm{~nm}$ model, resp.). 
heralding the formation of highly localized shear band along the shear plane [11].

In order to further understand the distribution of shear bands and the local strain along the decrease of model size during the deformation process, we use the local strain $\eta_{i}^{\text {Misses }}$ to monitor the difference of the deformation [12].

$\eta_{i}^{\text {Misses }}$ is measured by referenced to the relaxed glass prior to employing uniaxial tensile deformation along $z$-direction. The atoms in the Figure 4 are colored by the $\eta_{i}^{\text {Misses }}$ which tend to blue representing the smaller local strain value and tend to red representing larger local strain value.

From Figure 4(a) we can clearly see the shear band apparent on the direction of $45^{\circ}$ and $135^{\circ}$ and throughout the entire model, which can lead to shear instability with the increase of following strain. While Figures 4(b) and 4(c) present obvious transition process; $9 \mathrm{~nm}$ model appeared stress localization on the $45^{\circ}$ and $135^{\circ}$ direction, but without the formation of shear band, is a kind of transition to shear transformation process. The stress localization in $D=7 \mathrm{~nm}$ model is distributed scatteredly on the surface and inside with blurred direction and Figure 4(d) model only with several obvious localization on the surface, with the internal strain values almost uniformly distributed. This shows that the size has a very large effect on the deformation behavior of $\mathrm{ZrCu}$ MGs, with the decrease of the size, $\mathrm{ZrCu}$ MGs have experienced from the highly localized shear band formation to the homogeneous deformation, and the deformation process exists with obvious transition in $D=7-9 \mathrm{~nm}$ which demonstrate that the size decreased deformation mode is a gradual process instead of abrupt.

Statistic has been further performed on the individual atom along the $z$-direction strained $8 \%$ of the model and found that the number of atoms that is under the same shear strain accounts for different percentages of the total atoms in different models; the atoms that sustain shear strain larger than $8 \%$ in $3.6 \mathrm{~nm}$ model account for about $61 \%$ of the total atoms, while in $20 \mathrm{~nm}$ are only $37 \%$ and in $7 \mathrm{~nm}$ and $9 \mathrm{~nm}$ are about $55 \%$ and $49 \%$, respectively. To some extent, this illustrates that in the large size model, only a small fraction of the atoms are involved in deformation, while in small size model there are more atoms participating in deformation. We define those atoms as participating deformation atoms. How those atoms are distributed in the model is of great concern. So we divided each model into $0.1 \mathrm{~nm}$ along the loading axis and plot the average von Mises shear strain in Figure 5 as a function of position.

From Figure 5 we can find that the maximum shear strain of schematic atoms decreases along the decrease of model size. And in the model in Figures 5(a) and 5(b) there are about $57 \%$ and $35 \%$ atoms, respectively, gathered in tensile end and sustained larger shear strain, which is a kind of unstable distribution state. While in the model in Figures 5(c) and 5(d) those atoms are basically evenly distributed in the matrix model, sharing the strain caused by the plastic deformation, making the model a high tensile strength and plastic deformation ability.

Rearrangement would take place when local atomic strain beyond a certain critical threshold [13-15], which exhibits a strong autocatalytic behavior, is responsible for a considerable flow softening $[16,17]$. That is larger local atomic strain more propensity of strain localization and the formation of shear band. In our study we find that in the smallest model there are $61 \%$ atoms that participate in deformation; thus the strain assigned to individual atom will significantly decrease, which avoids large atomic rearrangement during the deformation process, so these atoms basically diffuse in the model matrix. While there are only $37 \%$ atoms involved in the deformation of the $D=20 \mathrm{~nm}$ model, the strain assigned to a single atom is greatly increased, so these atoms are more prone to restructuring. As a consequence, plastic deformation suffers a severe instability and forms the highly localized shear band. With the decrease of the model size, the fraction of atoms participating in deformation gradually increase, and this is the reason why the transition of the deformation mode is gradual instead of abrupt.

\section{Conclusion}

In summary, our $\mathrm{MD}$ simulations indicate that with the decrease of the model size the tensile strength of $\mathrm{ZrCu}$ metallic glass increases significantly and its deformation mode evolves from highly localized shear band formation to homogeneous deformation with obvious transition in $D=$ $7-9 \mathrm{~nm}$. We further find that when the models strained $8 \%$ along the $z$-direction there are $61 \%$ atoms involved in the deformation in the smallest model and due to avoiding large atomic rearrangement those atoms evenly distributed in the matrix. While only $37 \%$ atoms participate in deformation in the $D=20 \mathrm{~nm}$ model and because of an inevitable atomic rearrangement, the model exhibits a severe instability and forms the highly localized shear band.

\section{Conflict of Interests}

The authors declare that there is no conflict of interests regarding the publication of this paper.

\section{Acknowledgments}

This work was supported by the National Basic Research Program of China (2010CB731600) and the National Natural Science Foundation of China (51271161 and 51271162).

\section{References}

[1] E. Pekarskaya, C. P. Kim, and W. L. Johnson, "In situ transmission electron microscopy studies of shear bands in a bulk metallic glass based composite," Journal of Materials Research, vol. 16, no. 9, pp. 2513-2518, 2001.

[2] C. A. Schuh, T. C. Hufnagel, and U. Ramamurty, "Mechanical behavior of amorphous alloys," Acta Materialia, vol. 55, no. 12, pp. 4067-4109, 2007.

[3] D. Jang and J. R. Greer, "Transition from a strong-yet-brittle to astronger-and-ductile state by size reduction of metallic glasses," Nature Materials, vol. 9, no. 3, pp. 215-219, 2010. 
[4] C. A. Volkert, A. Donohue, and F. Spaepen, "Effect of sample size on deformation in amorphous metals," Journal of Applied Physics, vol. 103, no. 8, Article ID 083539, 6 pages, 2008.

[5] M. T. Myers, S. Charnvanichborikarn, C. C. Wei et al., "Phase transition, segregation and nanopore formation in high-energy heavy-ion-irradiated metallic glass," Scripta Materialia, vol. 67, no. 11, pp. 887-890, 2012.

[6] E. G. Fu, J. Carter, M. Martin et al., "Ion irradiation induced nanocrystal formation in amorphous $\mathrm{Zr}_{55} \mathrm{Cu}_{30} \mathrm{Al}_{10} \mathrm{Ni}_{5}$ alloy," Nuclear Instruments and Methods in Physics Research B: Beam Interactions with Materials and Atoms, vol. 267, no. 17, pp. 28272831, 2009.

[7] J. Carter, E. G. Fu, M. Martin et al., "Effects of Cu ion irradiation in $\mathrm{Cu}_{50} \mathrm{Zr}_{45} \mathrm{Ti}_{5}$ metallic glass," Scripta Materialia, vol. 61, no. 3 , pp. 265-268, 2009.

[8] C. Q. Chen, Y. T. Pei, O. Kuzmin, Z. F. Zhang, E. Ma, and D. Hosson, "Intrinsic size effects in the mechanical response of taper-free nanopillars of metallic glass," Physical Review BCondensed Matter and Materials Physics, vol. 83, no. 18, Article ID 180201, 4 pages, 2011

[9] D. Wang, Y. Li, B. B. Sun, M. L. Sui, K. Lu, and E. Ma, "Bulk metallic glass formation in the binary $\mathrm{Cu}-\mathrm{Zr}$ system," Applied Physics Letters, vol. 84, no. 20, pp. 4029-4031, 2004.

[10] S. Plimpton, "Fast parallel algorithms for short-range molecular dynamics," Journal of Computational Physics, vol. 117, no. 1, pp. $1-19,1995$.

[11] A. J. Cao, Y. Q. Cheng, and E. Ma, "Structural processes that initiate shear localization in metallic glass," Acta Materialia, vol. 57, no. 17, pp. 5146-5155, 2009.

[12] F. Shimizu, S. Ogata, and J. Li, "Theory of shear banding in metallic glasses and molecular dynamics calculations," Materials Transactions, vol. 48, no. 11, pp. 2923-2927, 2007.

[13] A. S. Argon and H. Y. Kuo, "Plastic flow in a disordered bubble raft (an analog of a metallic glass)," Materials Science and Engineering, vol. 39, no. 1, pp. 101-109, 1979.

[14] A. S. Argon, "Mechanisms of inelastic deformation in metallic glasses," Journal of Physics and Chemistry of Solids, vol. 43, no. 10, pp. 945-961, 1982.

[15] V. V. Bulatov and A. S. Argon, "A stochastic model for continuum elasto-plastic behavior. I. Numerical approach and strain localization," Modelling and Simulation in Materials Science and Engineering, vol. 2, no. 2, pp. 167-184, 1994.

[16] M. L. Falk and J. S. Langer, "Dynamics of viscoplastic deformation in amorphous solids," Physical Review E-Statistical Physics, Plasmas, Fluids, and Related Interdisciplinary Topics, vol. 57, no. 6, pp. 7192-7205, 1998.

[17] H. Bei, S. Xie, and E. P. George, "Softening caused by profuse shear banding in a bulk metallic glass," Physical Review Letters, vol. 96, no. 10, Article ID 105503, 4 pages, 2006. 

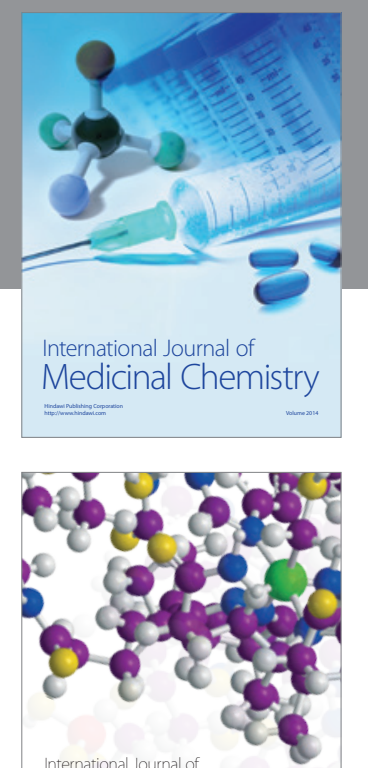

\section{Carbohydrate} Chemistry

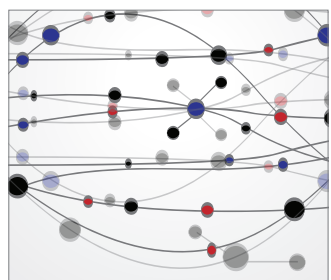

The Scientific World Journal
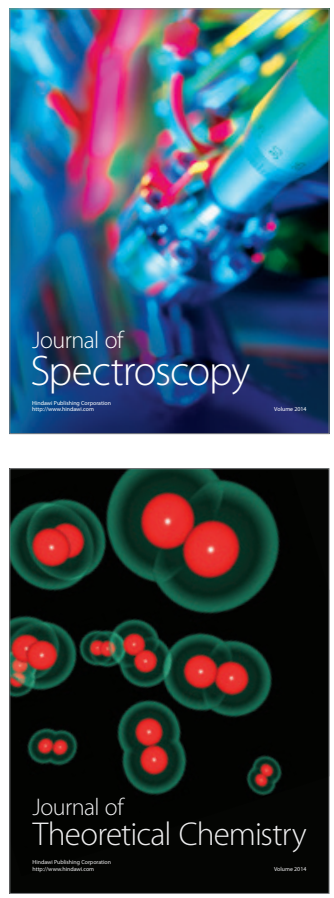
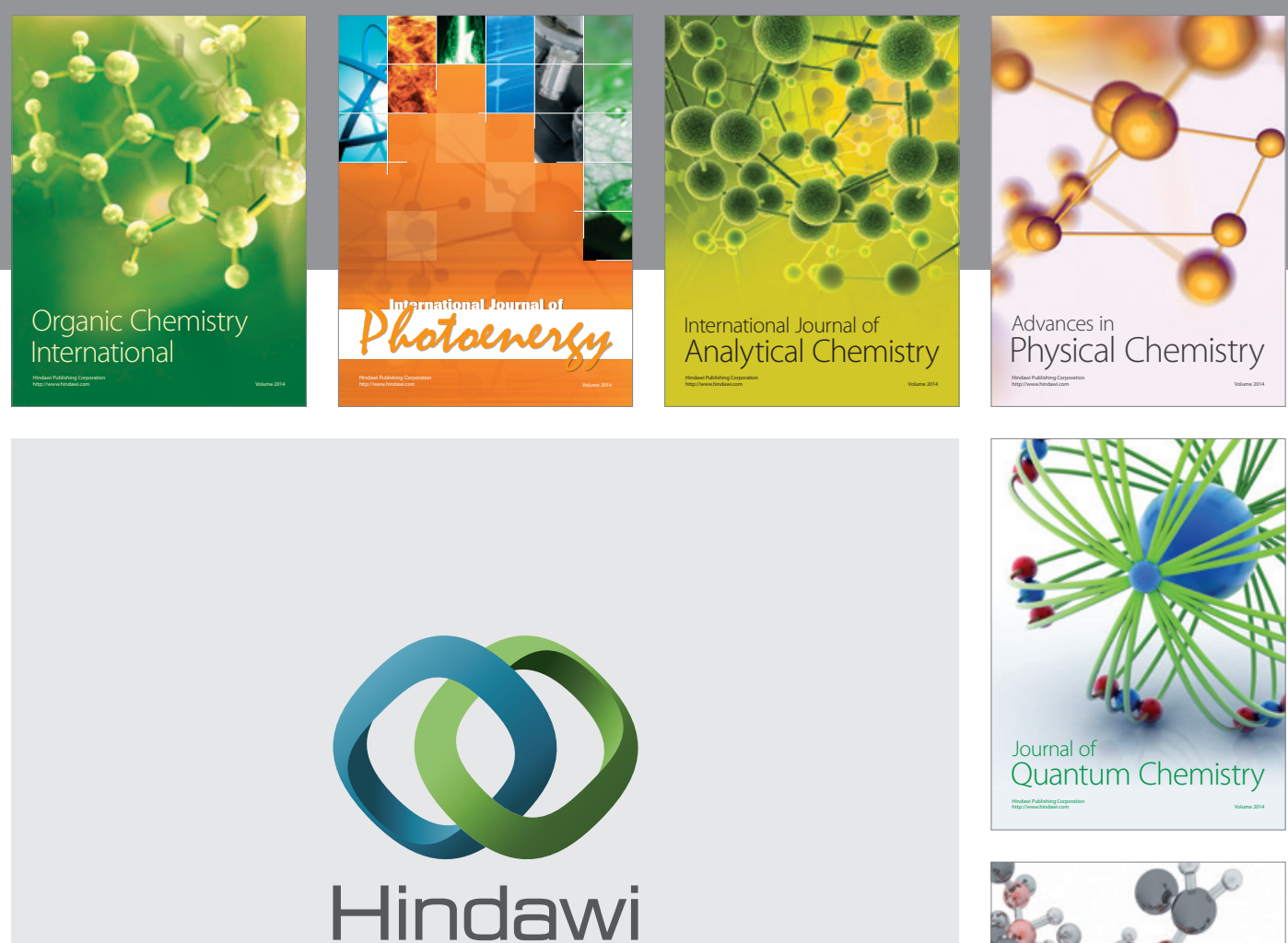

Submit your manuscripts at

http://www.hindawi.com

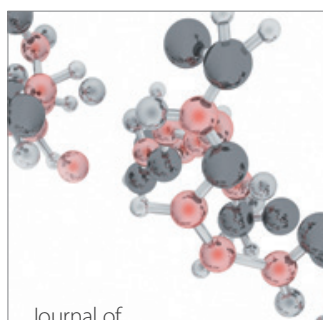

Analytical Methods

in Chemistry

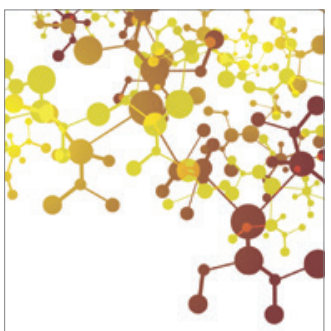

Journal of

Applied Chemistry

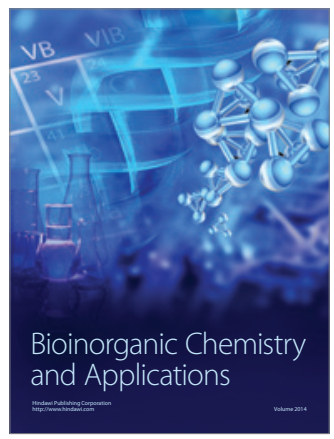

Inorganic Chemistry
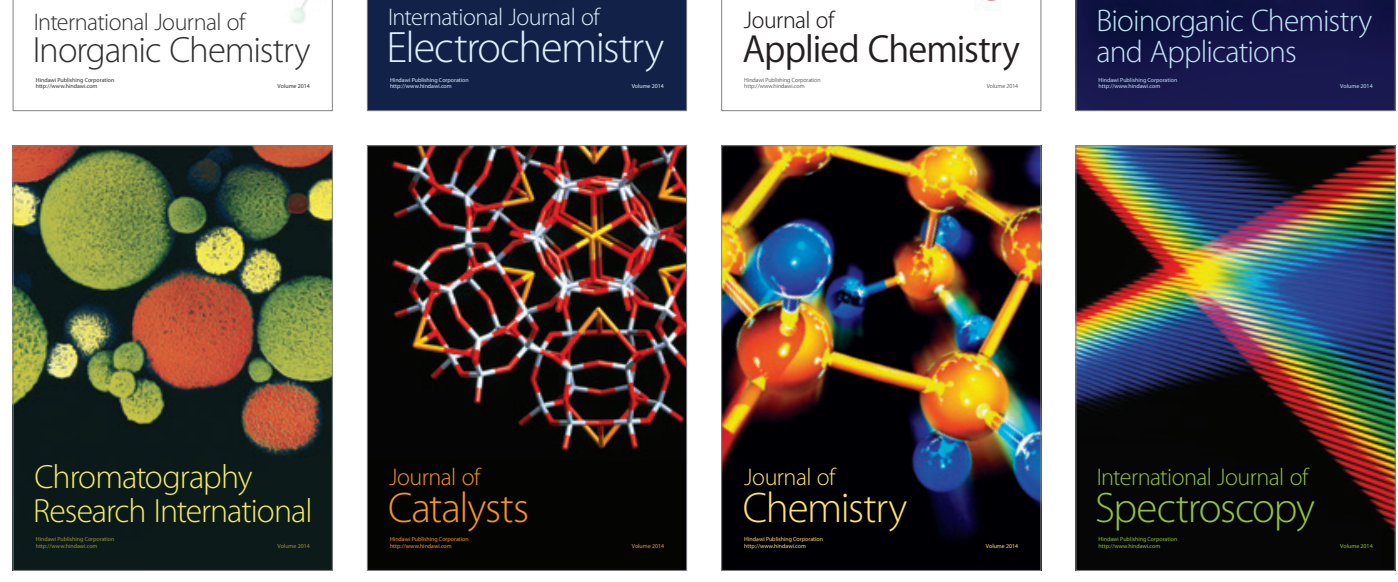